\title{
Study Design VI - Ecological Studies
}

\author{
Kate Ann Levin \\ Child and Adolescent Health Research Unit, University of Edinburgh, Edinburgh, Scotland, UK
}

Previously in this series I have given an overview of the main types of study design and the techniques used to minimise biased results. In this article I describe more fully ecological studies, their uses, advantages and limitations.

Evidence-Based Dentistry (2003) 7, 60-61. doi:10.1038/sj.ebd.6400454
An ecological study is an observational study defined by the level at which data are analysed, namely at the population or group level, rather than individual level. Ecological studies are often used to measure prevalence and incidence of disease, particularly when disease is rare. They are inexpensive and easy to carry out, using routinely collected data, but they are prone to bias and confounding. Also, because they are area-level studies, care must be taken when extrapolating either to individuals within the area level of measurement, or to a higher population level. Although other study designs are generally considered more reliable, particularly in the inference of causation, the population context of individual characteristics has been shown to be a stronger determinant of disease at population level than individual level risk factors.

\section{Why carry out an ecological study?}

An ecological study design is used when;

- the purpose of the study is to monitor population health so that public health strategies may be developed and directed;

- the purpose of the study is to make largescale comparisons, eg, comparisons between countries;

- the purpose of the study is to study the relationship between population-level exposure to risk factors and disease, or in order to look at the contextual effect of risk factors on the population;

- measurements at individual level are not available, eg. confidentiality might require that individuals are anonymised by aggregation of data to small area level; or

- the disease under investigation is rare, requiring aggregation of data for any analysis to be carried out.

Types of measurement in ecological studies In ecological studies health outcomes are aggregates of individual health data, eg: prevalence, incidence, rate of disease. Ecological risk or exposure data takes the form of one or more of the following:

- Aggregate measures; the data are summaries of individual level data eg, mean $\mathrm{dmft}$, percentage of children with no caries, area-level deprivation indices

- Environmental measures; equivalent individual level data are conceivable eg, mean annual exposure to fluoridation

- Global measures; there are no equivalent individual level data eg, number of dental practices, population density.

\section{Types of ecological studies}

Geographical; This type of study compares one geography with another by assessing the health of the population of each. Exposures for geographies may also be measured and included in analysis as well as other potential confounding variables such as demographic and socioeconomic information.

Longitudinal; A population is monitored to assess changes in disease over time. Again, confounding factors are often included in analysis.

Migration; Data of migrant populations are collected and analysed. The unit of interest is neither time nor place, but population type.

\section{The ecological fallacy}

The ecological fallacy is a type of confounding specific to ecological studies. It occurs when relationships which exist for groups are assumed to also be true for individuals. For example, an area with a majority of girls may have higher mean dmft than that of a second area with a majority of boys. The conjecture might therefore be that girls have higher dmft than boys. There are two types of potential confounding in this inference. The first is confounding which is common to all observational studies, i.e. because of the unknown characteristics of the areas being studied - for example, the first area might have higher deprivation than the second. The conjecture might therefore be that children from deprived areas have higher dmft. The second type of confounding is what is known as the 'ecological fallacy'. Even if all confounders are adjusted for, the aggregate nature of such a study tells us nothing about who in the population has high $\mathrm{dmft}$. Although there are only a few boys in the first population, perhaps these are the only children with dmft, albeit extremely high $\mathrm{dmft}$, bringing the mean above that of the second population. If this were true then our conjecture would be incorrect and may even be reversed at the individual level.

\section{Analysis of ecological data}

As with all observational studies, in order to overcome confounding, regression analysis is advisable. Multilevel modelling techniques have been developed, where analysis includes both individual and population level data, thus overcoming the ecological fallacy and enabling examination of contextual effects. Dental data are suited to multilevel modelling due to the clustered nature of disease, within surfaces, teeth, individuals, as well as group clusters such as schools, dental practices and geographical areas. Where individual level data are not known, for example where data are only available in an aggregated form, care must be taken in making causal inferences due to aggregation bias and the ecological fallacy.

\section{Recommended reading}

1. Pearce $N$. The ecological fallacy strikes back. I Epidemiol Community Health, 2000; 54: 326-327.

2. Piantadosi S, Byar DP, Green SB. The ecological fallacy. Am J Public Health, 1988; 127: 893-904.

3. Susser M. The logic in ecological: I. The logic of analysis. Am J Public Health, 1994: 84; 825-829.

4. Susser M. The logic in ecological: I. The logic of design. Am J Public Health, 1994: 84; 830-835. 